\title{
Optimisation of Flow Resistance and Turbulent Mixing over Bed Forms
}

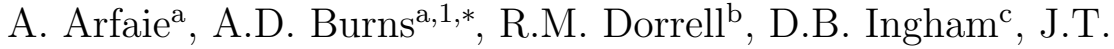 \\ Eggenhuisen $^{\mathrm{d}}$, W.D. McCaffrey ${ }^{\mathrm{e}}$ \\ ${ }^{a}$ School of Chemical and Process Engineering, University of Leeds, Leeds LS2 9JT, UK \\ ${ }^{b}$ Institute of Energy and Environment, University of Hull, Hull, HU6 7RX \\ ${ }^{c}$ Department of Mechanical Engineering, University of Sheffield, Sheffield, S10 2TN, UK \\ ${ }^{d}$ Department of Earth Sciences, Utrecht University, PO Box 80021, 3508 TA Utrecht, \\ Netherlands \\ ${ }^{e}$ School of Earth and Environment, University of Leeds, Leeds LS2 9JT, UK
}

\begin{abstract}
Previous work on the interplay between turbulent mixing and flow resistance for flows over periodic rib roughness elements is extended to consider the flow over idealised shapes representative of naturally occurring sedimentary bed forms. The primary motivation is to understand how bed form roughness affects the carrying capacity of sediment-bearing flows in environmental fluid dynamics applications, and in engineering applications involving the transport of particulate matter in pipelines. For all bed form shapes considered, it is found that flow resistance and turbulent mixing are strongly correlated, with maximum resistance coinciding with maximum mixing, as was previously found for the special case of rectangular roughness elements. Furthermore, it is found that the relation between flow resistance to eddy viscosity collapses to a single monotonically increasing linear function for all bed form shapes considered, indicating that the mixing characteristics of the flows are independent of the detailed morphology of individual roughness elements.
\end{abstract}

\footnotetext{
* Corresponding author.

Email address: a.d.burns@leeds.ac.uk (A.D. Burns)
} 
Keywords: Turbulent flow, Roughness, CFD, Bed forms

\section{Introduction}

Many industrial and environmental flows are subject to diminished or enhanced turbulence, and flow resistance due to presence of rough surfaces. In heat transfer applications, rib roughened surfaces are employed to enhance heat transfer characteristics in heat exchanger design [I]. Experimental and numerical studies have been performed to investigate the enhancement of heat transfer by the presence of roughness elements of a wide range of shapes [2, [3]. Furthermore, analytical and numerical optimisation studies have been performed in order to search for roughness shapes which optimise both heat transfer and friction loss performances [4].

Applications in the natural environment include the evolution of ribbed scales in sharks [5], and the formation of bed forms, such as dunes, in sediment carrying flows [6]. Previous work of Arfaie et al. [[, , 8] has shown that there are optimal patterns of large-scale roughness elements, such as ribs, to maximise turbulence or to minimize flow resistance. This conclusion apparently supported the work of Eggenhuisen and McCaffrey [9], who proposed that run-out lengths of particulate gravity currents are enhanced by the presence of rugose bed forms on the ocean floor, such as scours and dunes. Eggenhuisen and McCaffrey conducted gravity current experiments where the flow was perturbed by the presence of a single rectangular roughness element. They observed that the profiles for vertical turbulent normal stresses showed enhanced mixing compared to unperturbed turbulence profiles and concluded that enhanced mixing via bed forms result in a net distribution of sediments towards the upper region of the flow, and thus a reduction in density stratification and an increase in the run-out distance of the flow.

Turbidity current run-out length is controlled by the balance of potential to kinetic energy conversion as a function of the rate of energy dissipation through drag, diffusion and viscous dissipation. The potential energy 
is controlled by the balance of turbulent particle diffusion with gravitational settling. Thus maximizing flow turbulence is expected to increase the eddy diffusivity of particles, the potential energy of the flow and hence promote greater run out [10-13].

Arfaie et al. [7] sought to find further support for this hypothesis by performing a series of numerical investigations to study the effect of lower boundary roughness on turbulent flow in a two-dimensional channel. Periodic arrays of rectangular roughness elements were considered over a wide range of roughness spacing to height ratios, $w / k$. Computations of volume averaged eddy viscosity were performed over this range in order to establish the optimum spacing that produces maximum turbulence enhancement and mixing. This was found to occur when $w / k$ is approximately equal to 7. Moreover, this value is only weakly dependent on Reynolds number, and the decay rate of turbulence enhancement as a function of $w / k$ beyond the optimum spacing is slow. In addition to this, computations of friction factor as a function of $w / k$ indicated that maximum resistance to flow also occurs at the same value of $w / k=7$. Consequently, the implications on particulate gravity current run-out length were inconclusive, as optimized turbulence mixing tends to act to keep particles in suspension, hence increasing run-out length, whilst optimized resistance acts as a large drain on turbulence kinetic energy, hence decreasing run-out length.

The purpose of this paper is to extend the work of Arfaie et al. [ [ ] to consider the flow over idealised bed form shapes encountered in geophysical flows, such as dunes, anti-dunes, and symmetric triangular bed forms. This builds on work by McLean et al. [14] who constructed semi-analytical models based on boundary layer theory for velocity profiles, and boundary shear stresses for flows over two-dimensional dunes. They emphasized the importance of splitting the total drag force on the bed into the pressure drag, or form drag, and the viscous drag, or skin friction. The former is largely responsible for draining the flow of mean kinetic energy, whilst the latter 
is largely responsible for erosion of particles from the bed into suspension, hence increasing potential energy. Subsequent developments are reviewed by Best [6] in the context of dunes in rivers. Furthermore, similar CFD research has been carried out to model air flows and sediment transport over aeolian dunes [15-18] and air pollution dispersion over urban canyons [19-21]. We perform computational fluid dynamics (CFD) calculations to obtain detailed information on the flow over a variety of idealized two-dimensional dunes. The results are then used to obtain information on the resistive drag and turbulence mixing characteristics as a function of dune shapes and aspect ratios, with particular emphasis on the correlation between flow resistance and turbulent mixing for the different shapes.

In addition to the environmental flow applications considered here, dunes deposited from suspended particles have a significant effect on the flow resistance and sediment carrying capacity of engineering conduits and pipelines, such as those employed in sewage systems [22-24]. May [22] reported on experimental results and models for the effects of a wide range of dune types deposited by non-cohesive particles in sewage systems, noting that their effects were indeed significant, as minimum slope and flow velocities need to be specified in order for sewers to become self-cleansing. This work was extended to cohesive particles by Skipworth et al. [23]. Coleman et al. [24] performed experiments that indicated that dunes deposited in closed conduits have much in common with dunes formed under free surface flows.

\section{Methods}

\subsection{Numerical Method}

Simulated flow over complex bedforms was modelled using the numerical method employed by Arfaie et al. [7]. Further details may be found in the $\mathrm{PhD}$ thesis of Arfaie [8]. Arfaie et al used a commercial CFD code ANSYS CFX-14.0 [25] to compare the predictions of a wide range of Reynolds Averaged Navier-Stokes (RANS) based turbulence models with the experimental 
results of Djenidi et al. [26] and the numerical Large Eddy Simulations of Cui et al. [27]. The standard $\mathrm{k}-\varepsilon$ model [28], used herein, was found to give adequate predictions of flow velocity profiles, and to provide the best compromise between accuracy and computational efficiency in order to permit the exploration of a wide parameter space.

\subsection{Idealised Bedforms}

The rib roughness elements studied by Arfaie et al. [7] are generalised to idealised shapes, similar to those studied by Moon et al. [2]. The shapes considered here are intended to represent bed forms occurring in the natural environment, see figure $\square$. The shapes $S 1$ are the rectangular roughness elements considered by Arfaie et al. [7]. Shapes $S 2-S 4$ represent symmetric bed forms, anti-dunes and dunes respectively. Shapes $S 5-S 7$ combine the simple shapes $S 1-S 4$ to approximate more realistic natural lower boundary rugosities of symmetric dunes, anti-dunes and normal dunes, respectively. Arfaie et al. [7] employ the categorisation of Perry et al. [29] of roughness elements into two distinct types of roughness, namely, $d$-type and $k$-type, where $d$ and $k$ denote channel height and roughness height, respectively. For a sufficiently low width-to-height ratio, $w / k \lesssim 2$, the flow undergoes a

skimming flow regime, in which there is little interaction from the vicinity of the roughness element to the outer flow region. This is known as $d-t y p e$ roughness. The $k$-type roughness regime is associated with $w / k \gtrsim 4$, where the flow in the roughness cavity begins to interact with the main body of the flow. See Jimenez [30] for a detailed discussion of $d$-type and $k$-type roughness regimes. Here, the roughness element height is denoted by $h$, and we perform a study of the effect of axial roughness length to height ratio $(c / h)$. The upper flat surface length of the roughness element $c$ is varied while the roughness element height is held fixed to cover aspect ratios $c / h=1,2,4$ and 8 for each of the bed geometries. The width-to-height ratio $w / h$ is kept fixed at the value $w / h=7$ which was determined by Arfaie et al. [7] to give optimal turbulent mixing and flow resistance for rectangular roughness 
elements. We have not established that this is optimal for all the shapes considered here; it is kept fixed in order to reduce the number of simulations that need to be performed.

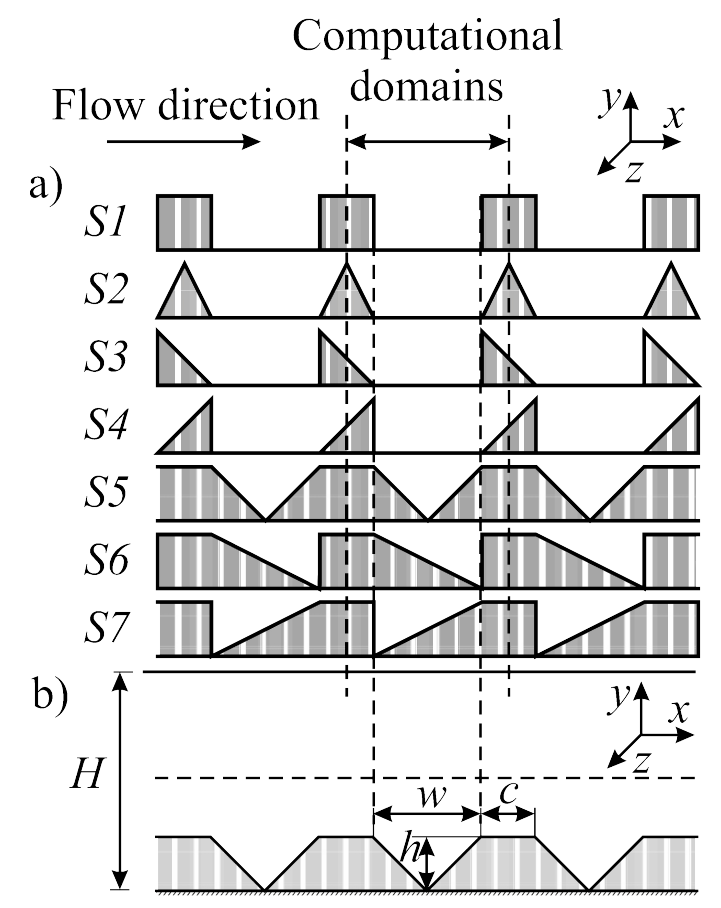

Figure 1: (a) Schematic illustration of the computational domains shown by the dashed lines for pressure driven flow over idealized bedforms under a periodic condition. (b) Channel flow configuration with roughness segment S5 positioned at the solid bed.

\subsection{Model Assumptions}

A shear flow of pure seawater over the idealised bed forms is considered. It is assumed that the particle concentrations are sufficiently small that they do not have a significant influence on the flow field. There is strong evidence that this configuration also gives insight into behaviour of dilute sediment gravity flows over bed forms; the velocity profiles of such flows in the inner wall region (below peak velocity) resemble those in fully developed channel flows (Kneller et al. [3]], Eggenhuisen and McCaffrey [9], figure Q). 


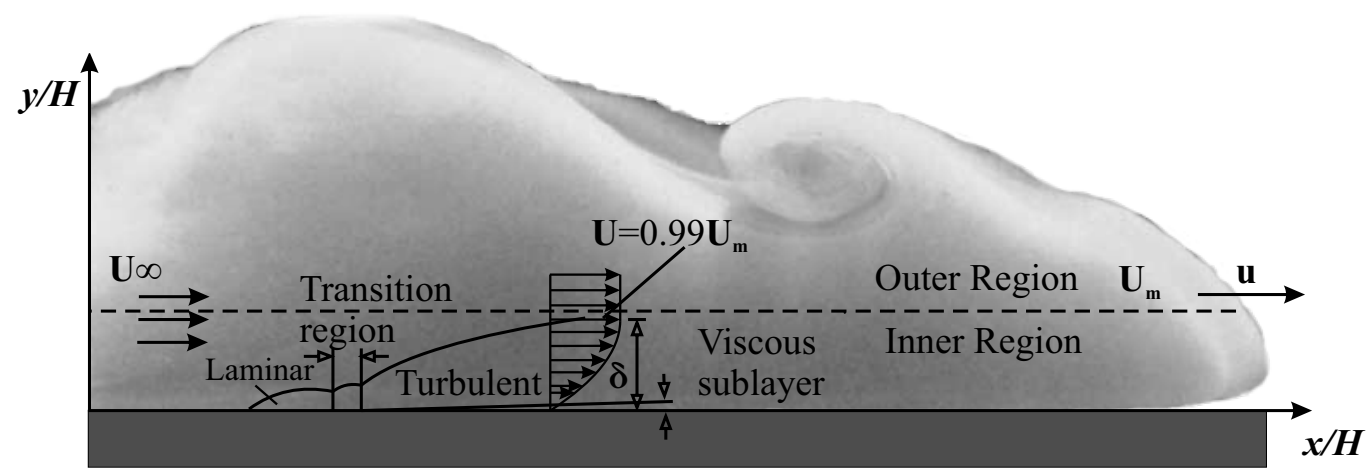

Figure 2: The structure of turbidity currents modified from the work by Kneller et al. [31] and Simpson [32]. Note: the velocity profile below the velocity maximum approximates that of a shear flow.

Numerical simulations were conducted for fully-developed turbulent flow

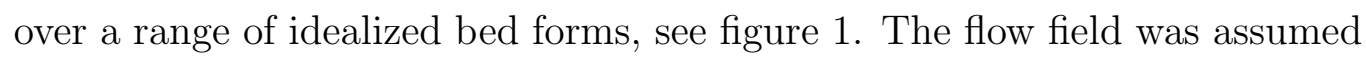
to be periodically repetitive in the stream-wise direction. Consequently, the computational domain was restricted to describe the flow over a single roughness element, as shown in figure 1 (a). The lower boundary was comprised of a hydraulically rough bed of one of forms $S 1-S 7$, whilst the upper boundary was flat, with no-slip boundary conditions imposed on both upper and lower boundaries. The flow was driven by applying a constant mean pressure gradient $\overline{\partial p / \partial x}$ added as a source term in the $x$-momentum equation. The derived periodic pressure field therefore represents fluctuations about a mean pressure field which is linear in the $\mathrm{x}$-direction. The flow was of density $\rho=1027 \mathrm{~kg} \mathrm{~m}^{-3}$ and kinematic viscosity $\nu=1.36 \times 10^{-6} \mathrm{~m}^{2} \mathrm{~s}^{-1}$, typical of seawater. A value of $\overline{\partial p / \partial x}=0.5 \mathrm{~kg} \mathrm{~m}^{-2} \mathrm{~s}^{-2}$ was chosen to achieve a high friction shear Reynolds number $R e_{\tau} \approx 10^{5}$, where $R e_{\tau}=\left(u_{\tau} H / 2\right) \nu$ is based on the shear velocity $u_{\tau}=\sqrt{H /(\rho(\overline{\partial p / \partial x)}}$ and half-channel height $H / 2$. This was motivated by the findings of Arfaie et al. [G] that turbulent flow over rugose bed forms is only weakly dependent upon Reynolds number. 


\section{Results and Discussion}

\subsection{Velocity Fields}

Here we analyze how the various idealized bed form shapes modulate the flow velocity field. Velocity magnitude contours are plotted as a function of bed form topography and aspect ratio. Shapes 1-4 in figure B behave as $k$-type roughness elements as the cavity length $w$ is larger than the height $h,(w / h=7)$. The $d-$ type surfaces $(S 5-S 7)$ in figure $⿴$ are arguably closer to the flat wall case and consequently are less effective at slowing down the flow than the $k$-type surfaces. Therefore, wall shear stress is greater in $d$-type bed forms rather than $k$-type bed forms.

Small secondary recirculating regions are observed behind the downstream edge of shape $S 1-S 4$ and $S 7$. The structure of these vortices depends significantly on the fluid-facing slope angle. For example, shapes $S 1$ and $S 4$ with downstream edges at 90 degrees to the horizontal produce larger vortices compared to shape $S 2$, whilst shape $S 5$ has no recirculation region at all. Comparison of streamlines for shapes $S 3$ and $S 6$ indicates that the development of the secondary vortex on the slope facing bed forms does not depend significantly on the spacing between two bed forms. The flow streamlines in figure 3 show that, for $k$-type roughness, the flow separates near the bed form crest, followed by reattachment of the flow on the flat bed next to the adjacent bed forms.

Moreover, the streamlines of shape $S 1$ show that as the aspect ratio $c / h$ increases, the focal point of the recirculation region shifts more towards the leading face of the downstream rib, approaching intermediate type roughness behavior. This behavior is also apparent for shape $S 4$ where the streamlines become more parallel as aspect ratio values increase from 1 to 8 . 
Velocity Magnitude: k-type roughness elements

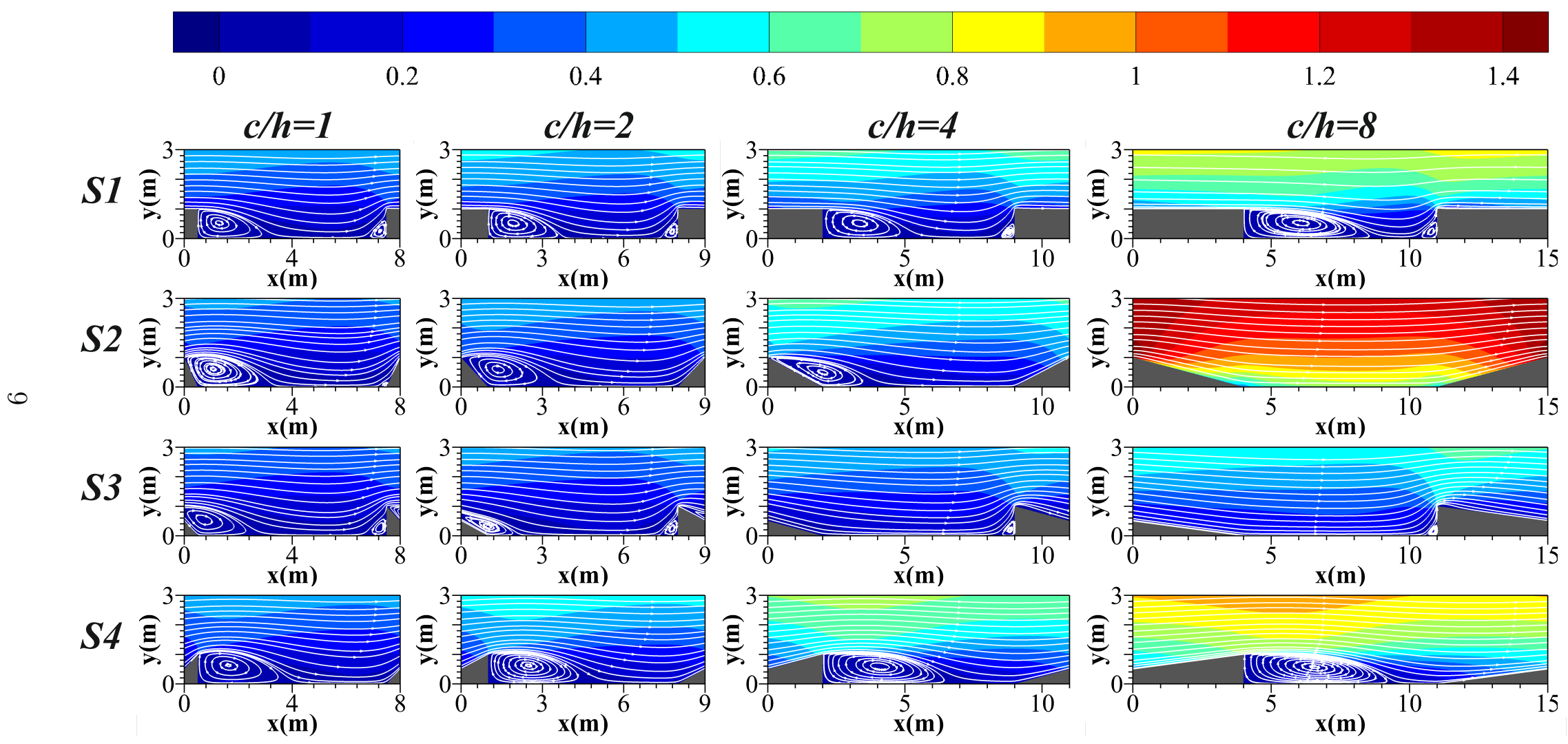

Figure 3: Matrix view of the velocity magnitudes with flow streamlines over $k$-type bed forms with varying aspect ratio $c / h$. 
Velocity Magnitude: d-type roughness elements

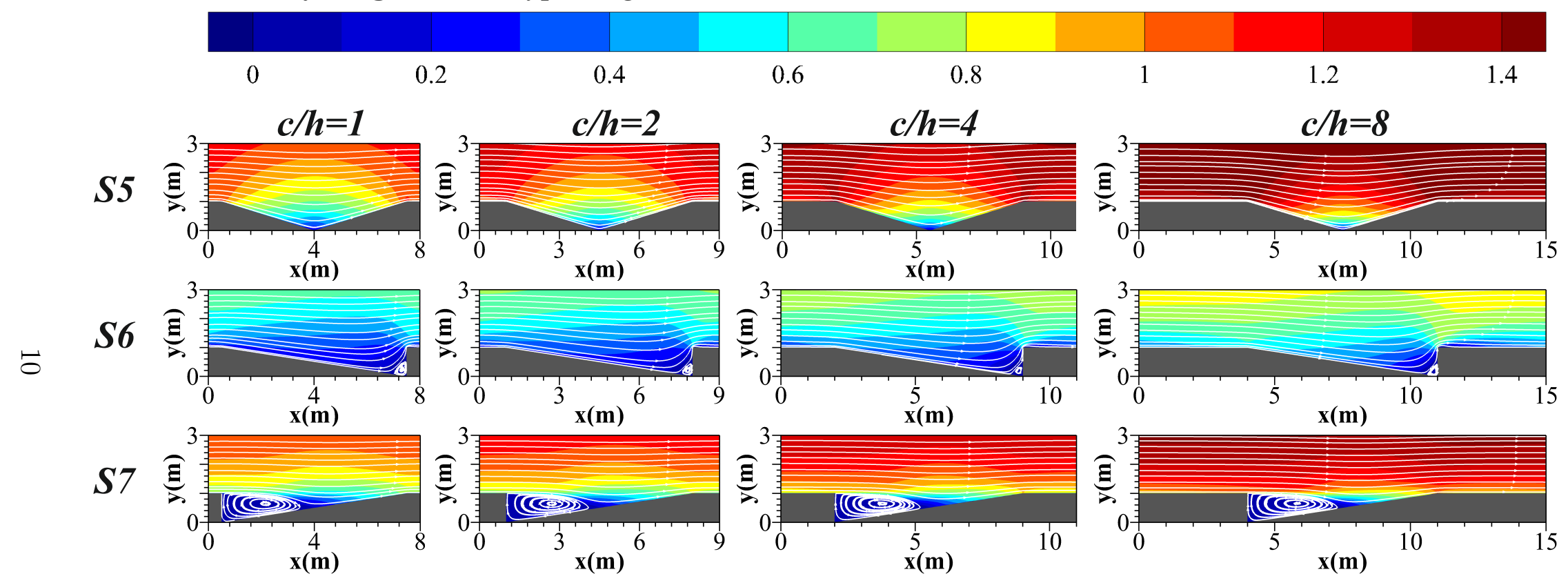

Figure 4: Matrix view of the velocity magnitudes with flow streamlines over $d$-type bed forms with varying aspect ratio $c / h$. 


\subsection{Mean Flow and Flow Resistance}

\subsubsection{Mean Flow}

In comparison to a flat bed, the presence of bed forms modulates the flow. Both figures 3 and 4 show that as flow obstruction and thus frictional drag is reduced, i.e. as aspect ratio $c / h$ increased, the mean axial flow velocity increases significantly. Also, the re-attachment length increases for all $k$-type bed forms with the increase in aspect ratio. The behavior in reattachment is attributed to shallowing of the lee slope reducing turbulence intensity and diffusivity in the space between the recirculation region and the next roughness element.

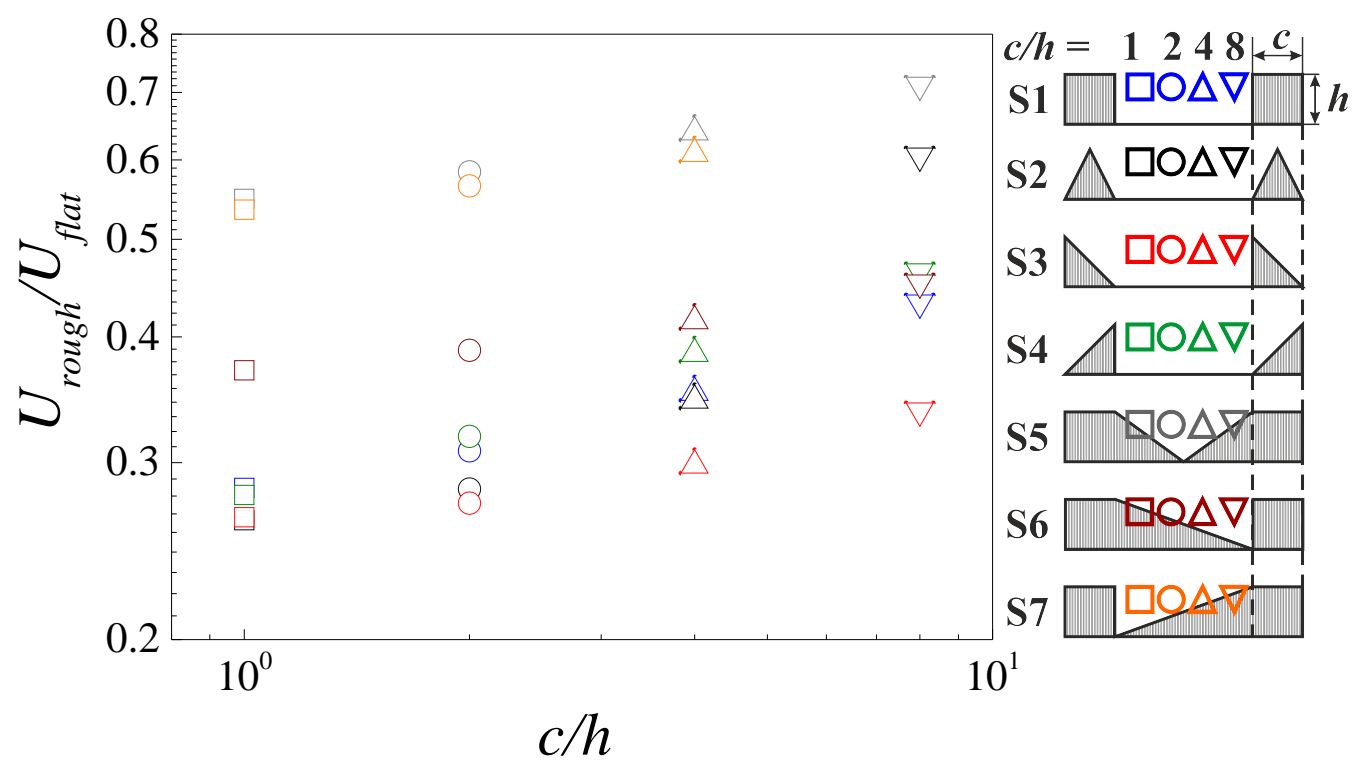

Figure 5: Normalised stream-wise mean velocity as a function of aspect ratio for all bed roughness cases.

The cross-sectionally averaged stream-wise velocity for flow over bed forms is denoted by $U_{\text {rough }}$. The corresponding quantity for the flat bed case is denoted by $U_{\text {flat }}$, and we use this to normalise the averaged velocity in the rough bed cases. The ratio $U_{\text {rough }} / U_{\text {flat }}$ is plotted against aspect 
ratio $c / h$ for all lower roughness boundaries in figure 5. Due to obstruction of the flow by bed roughness elements $U_{\text {rough }} / U_{\text {flat }}$ is strictly less than unity. The maximum average velocity across all aspect ratios is seen for the smoothed roughness element $S 5$, reducing flow separation, and the minimum for pointed roughness element $S 3$, enhancing flow separation.

Figure 5 indicates that shape $S 2$ has a greater gradient in velocity with respect to change in aspect ratio compared to that of other bed shapes. This result agrees with the DNS results of Orlandi et al. [3] in which a higher average flow velocity is seen over triangular bars compared to square bars. In addition, there is an increase in velocity between the $d$-type and the $k$-type flows. However, the magnitude of this shift decreases with the increase in aspect ratio. The value of $U_{\text {rough }} / U_{\text {flat }}$ in the idealized asymmetric dune $S 4$ is greater than that of the idealized symmetric dune $S 2$.

\subsubsection{Flow Resistance}

As all simulations are performed under a fixed imposed pressure gradient, the results described above on variation of mean velocity with shape and aspect ratio are a consequence of the variation of resistance to flow as these quantities are varied. The resistance to flow over the various bed geometries is computed using the Darcy-Weisbach friction factor $f$ defined in equation (四), based on the specified mean pressure gradient $\partial p / \partial x$ and the averaged stream velocity $\bar{U}$

$$
f=\frac{(H / 2)(-\partial p / \partial x)}{0.5 \rho \bar{U}^{2}}
$$

Figure [ 6 plots friction factor against aspect ratio of each of the bed form shapes. The results show that the friction factor decreases with increasing aspect ratio for all bed form shapes. The maximum flow resistance occurs for the transport of fluid over shape $S 3$ and the minimum flow resistance occurs for shape $S 5$. The friction factor is greater for $k$-type (with spacing) roughness elements than $d$-type (without spacing) cases. 


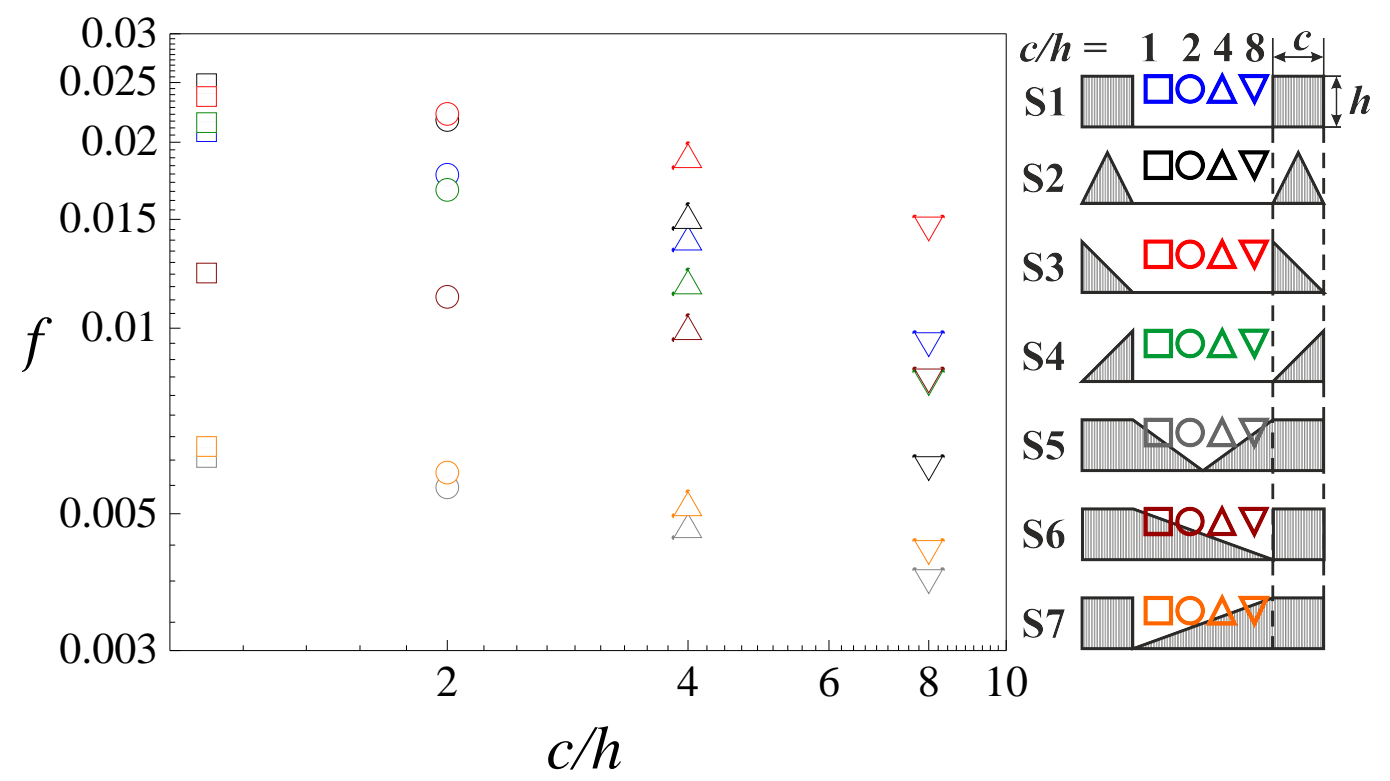

Figure 6: Darcy-Weisbach friction factor as a function of aspect ratio for flows over different roughness elements.

\subsubsection{Skin Friction and Form Drag}

In order to understand the dynamics of natural flows over bed forms, it is necessary to partition the total shear stress at the bottom boundary into the separate components of skin friction and form drag [14]. The skin friction is the component due to tangential stresses (viscous forces), and this is a determining factor for the rate of entrainment of sediment from the bed into the main flow. The form drag is the component due to normal stresses (pressure forces). For flow over blunt roughness elements, this is usually the dominant contributor to the overall drag force, due to flow separation generating large differences in pressure between the front and the back of the element.

The skin friction and form drag are determined by integrating the viscous and pressure forces along the wall, as described in equations (ㅈ) and (3) 
respectively

$$
\begin{gathered}
\vec{F}_{v}=\int_{w} \tau_{w} \vec{s} \cdot \vec{x} d s \\
\vec{F}_{p}=\int_{w} p \vec{n} \cdot \vec{x} d s
\end{gathered}
$$

The relative change in the skin friction and form drag are determined by non-dimensionalising the friction components (《) and (B) with the force applied to the lower boundary,

$$
\begin{aligned}
& D_{s}=\frac{\left|\vec{F}_{v}\right|}{1 / 2 \rho u_{f}^{2} A_{w}} \\
& D_{f}=\frac{\left|\vec{F}_{p}\right|}{1 / 2 \rho u_{f}^{2} A_{p}}
\end{aligned}
$$

Here $u_{f}$ is the area-weighted average velocity $u$ of the fully developed flow at the outlet of the flat bed case. The skin friction (⿴囗十) employs the wetted areas $A_{w}$, which for aspect ratios $c / h=1,2,4,8$ are given by $A_{w}=$ $9,11,15,23 \mathrm{~m}^{2}$ respectively. The form drag (eqn. 5) employs the projected area $A_{p}$, which is equal to $1 \mathrm{~m}^{2}$ for all bedform simulations.

Figure $\square$ plots absolute values of the form drag against skin friction for all bed form shapes and aspect ratios. Both skin friction and form drag are found to increase with increase in aspect ratio for all bed form shapes. The increase is due to the integral of the pressure and viscous forces for the extended surface produced by the increase in aspect ratio $c / h$. Further, figure $\square$ indicates that the value of skin friction is greater for $d$-type than for $k$-type bed forms. This is primarily due to either the lack of flow separation ( $S 5$ and S6), or flow separation with reattachment on the downstream sloped edge next adjacent element $(S 7)$ leading to a low pressure gradient. Consequently the value of form drag is greater for the $k$-type than the $d$-type bed forms. 


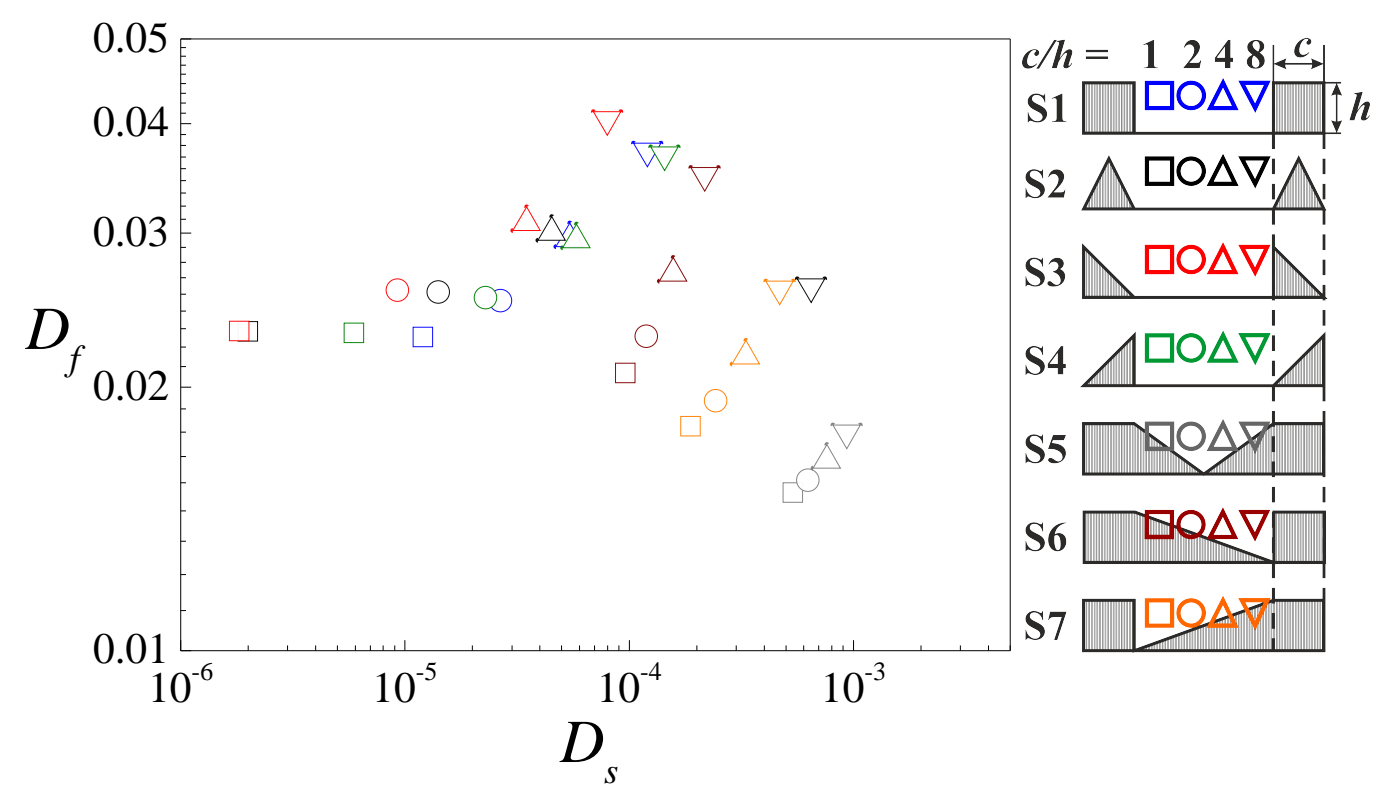

Figure 7: Form drag versus skin friction for all bed forms and aspect ratios.

\subsection{Turbulent Mixing}

Arfaie et al. [7] measured the amount of turbulent mixing in a periodically rectangular rib roughened channel using the non-dimensionalised eddy viscosity $\mu^{+}$,

$$
\mu^{+}=\frac{\mu_{t}}{\rho \bar{U}(H / 2)}
$$

Here we use $\mu^{+}$to evaluate the turbulent mixing potential of different bed geometries. Figure $\nabla$ plots the average value of $\mu^{+}$as a function of $c / h$ for all bed geometries. The overall results suggest that bedforms with high flow resistance also result in high turbulent mixing. The data further shows that $\mu^{+}$is reduced as the aspect ratio of the bed forms is increased. The lowest turbulent mixing occurs for bed form shapes with closed spacing (S5-S7) and

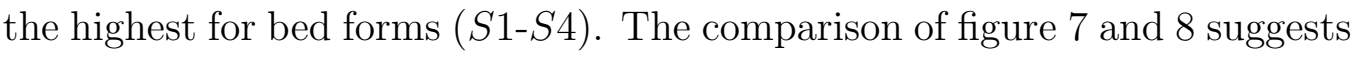
that turbulent mixing depends more on form drag than skin friction. 


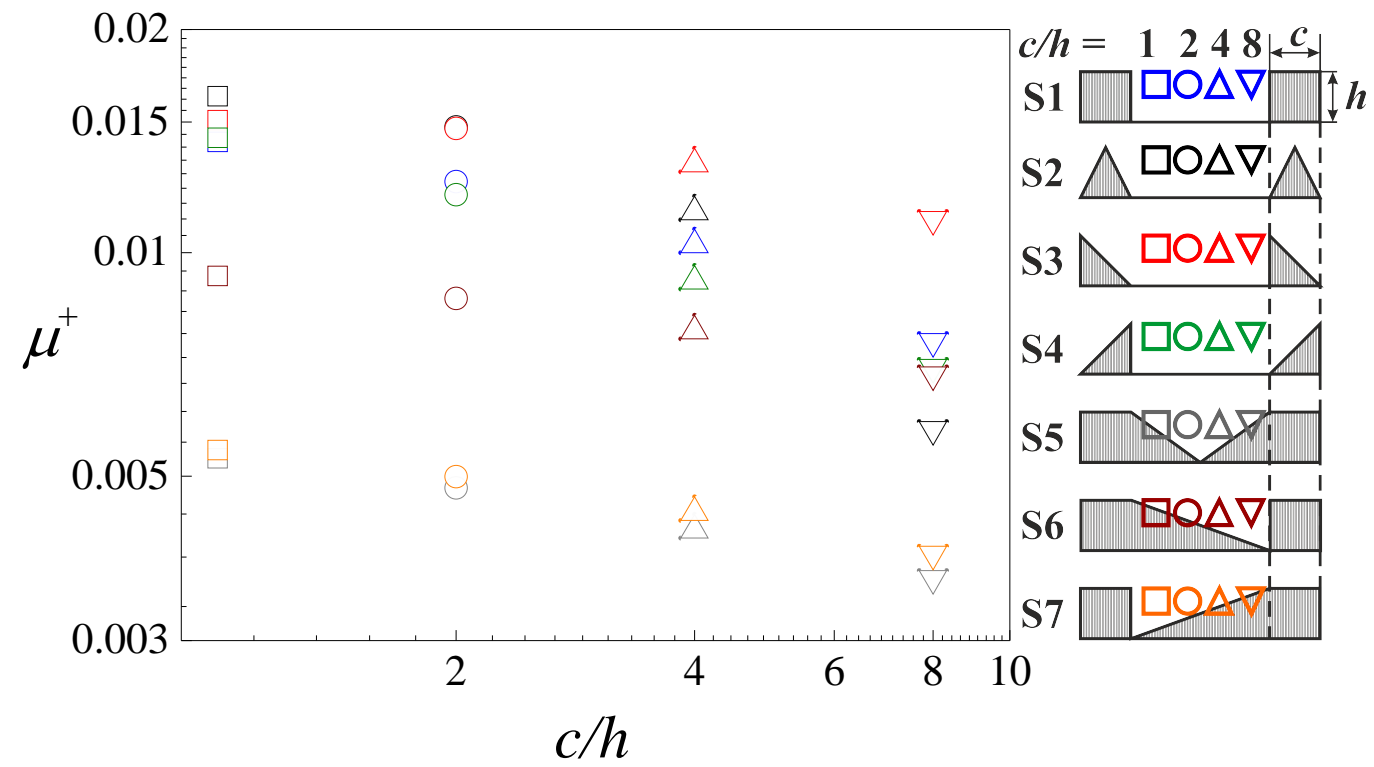

Figure 8: Normalised eddy viscosity as a function of aspect ratio $c / h$.

An important conclusion that can be made is that the strong correlation between turbulent mixing and flow resistance found by Arfaie et al. [7] for rectangular bedforms is also seen for the full range of bed form shapes considered here. Figure $\mathbf{9}$ plots the non-dimensionalised measures of flow resistance (friction factor $f$ ) and turbulence mixing (normalized eddy viscosity $\mu^{+}$) against each other for all bedform shapes and aspect ratios considered here. Significantly, the data effectively collapse onto the same straight line for all bed form shapes. This result is reminiscent of the observation that the friction factor for turbulent flow in a rough conduit correlates well with flow Reynolds number and roughness height, but is apparently independent of roughness morphology at least up to $5 \%$ relative roughness [33]. Figure 9 indicates that this degree of universality may extend to the larger roughness elements considered here.

Shape $S 5$ produces the least flow resistance and mixing. The low value of vertical mixing in this case appears to be caused by the lack of flow sep- 


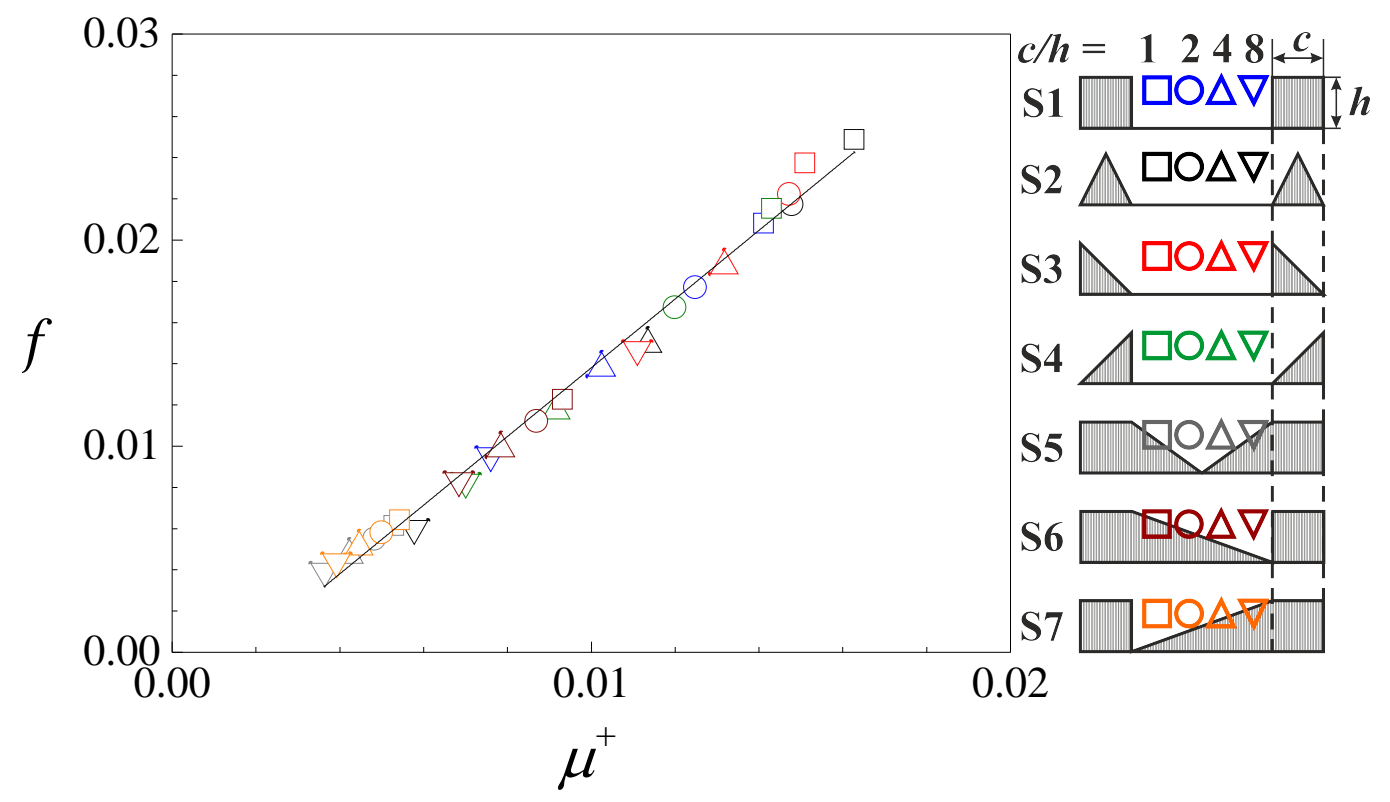

Figure 9: Friction factor as a function of normalized eddy viscosity for all bedforms.

aration and the closed gap between two adjacent bed forms. Moreover, the results indicate that flow separation has an insignificant effect on the value of upward sediment mixing for the skimming flow type $(S 5-S 7)$, where the flow reattaches on the slope of the downstream roughness element. Figure 9 further illustrates that the blunt facing bed forms $S 5$ and $S 7$ achieve a higher turbulent mixing than the slope facing bed form $S 6$. $k$-type surfaces also show an increase in $\mu^{+}$and $f$ with the orientation of the downstream facing flow (or stoss side). For instance, shapes $S 2$ and $S 4$ lead to reductions in the values of mixing and flow resistance compared to shape $S 1$ and $S 2$ (with $90^{\circ}$ side facing flow angle). For the $k$-type surfaces the effect of flow separation on the flow resistance and turbulent mixing is also weak. This effect is described for shape $S 3$ at $c / h=2$ (with flow separation) and $c / h=4$ (with absence of flow separation) in figure 3 . 


\subsection{Discussion}

The rate of sediment entrainment is a function of the skin friction. Therefore figure $\square$ predicts that the sediment entrainment rate is greater for bed forms $S 5-S 7(d-$ type $)$ than for $S 1-S 4$. The results also predict that the suspension of the sediment should be greater for $k$-type than for $d$-type surfaces. Both the sediment suspension and entrainment rate are predicted to increase with the decrease in slope angle.

Finally, in accord with the results of Arfaie et al. [7] we cannot conclude that any of the shapes considered definitively promote or reduce run out length, due to the subtle interplay that occurs between the competing effects of flow resistance and turbulent mixing on the overall kinetic and potential energy budgets.

Note that this work has considered integrally averaged measures of flow resistance and turbulent mixing for flows of pure liquid, neglecting details of particle transport. The results suggest that, in order to elucidate further the effects of bed form roughness on the energy budget of sediment-bearing flows, such as turbidity currents, further work is needed that investigates the details of particle concentrations, sedimentation and flow capacity. This will be the subject of further investigations.

\section{Conclusions}

Numerical simulations have been performed at a high Reynolds number for shear flow over a series of lower boundary roughness elements comprising a range of idealized bed form shapes, of varying crestal length to height ratio $c / h$ at a fixed width to height ratio $(w / h)$. The total basal shear stress is split into skin friction and form drag, with sediment entrainment proportional to skin-friction and turbulent mixing proportional to the sum of skin friction and form drag. Here we show how the respective magnitudes of these friction components vary as a function of scale of various bed form roughness shapes, with both $d$-type and $k$-type characteristics. Sediment erosion is expected 
to be greatest in the $d$-type bed forms where skin friction is observed to be largest. The results also demonstrate how bed forms affect the balance of energy lost (through frictional effects) as a function of the potential energy gained (through turbulent mixing).

The results indicate that, for all bed form shapes considered, both the friction factor and the dimensionless eddy viscosity decrease with increasing aspect ratio. Thus, flow resistance and turbulent mixing are strongly correlated, with maximum resistance coinciding with maximum mixing for all shapes considered. Figure $\mathbb{Q}$ indicates that the relation between flow resistance to eddy viscosity collapses to a single monotonically increasing linear function for all bed forms considered. This result is significant, as it supports the conjecture that the relations between frictional and mixing characteristics of the flows that we have considered are independent of the detailed morphology of the individual roughness elements.

\section{Software availability}

Software: ANSYS-CFX

Programming languages: Fortran 90/77 and CEL

Discretization technique: Finite volume

Current version: 14.0

Availability: Download definition file from https://drive.google.com/ drive/folders/1XHNEXIlzCivBDtAMycox1QRV--d_BB5D?usp=sharing

Operating system: Linux and Windows

License: Commercial

HPC: Dual socket with quad-core Intel X5560 (2.8GHz) processors per server; 12GB of DDR3 $1333 \mathrm{MHz}$ memory per server, 24GB flash disk and QDR Connect-X infiniband (ARC1 University of Leeds).

Parallel Run Mode: MPI (8 nodes and 8 processes per node used)

Contact address: ANSYS UK Ltd, Abingdon, United Kingdom

Telephone: +44 (0) 8000480462 
Fax: None

Email: support-uk@ansys.com

Web page: https://www.ansys.com

Year First available: 1991

Plotting tool: Tecplot https://www.tecplot.com/

\section{Acknowledgements}

This research was funded by the Turbidites Research Group industry consortium (Anadarko, BG, BHP Billiton, BP, ConocoPhillips, Maersk, Marathon, Nexen, Statoil, Tullow and Woodside.)

\section{References}

[1] R. Webb, E. Eckert, Application of rough surfaces to heat exchanger design, International Journal of Heat and Mass Transfer 15 (1972) 16471658.

[2] M.-A. Moon, M.-J. Park, K.-Y. Kim, Evaluation of heat transfer performances of various rib shapes, International Journal of Heat and Mass Transfer 71 (2014) 275-284.

[3] P. Orlandi, D. Sassun, S. Leonardi, Dns of conjugate heat transfer in presence of rough surfaces, International Journal of Heat and Mass Transfer 100 (2016) 250-266.

[4] H.-M. Kim, K.-Y. Kim, Shape optimization of three-dimensional channel roughened by angled ribs with rans analysis of turbulent heat transfer, International Journal of heat and mass transfer 49 (2006) 4013-4022.

[5] T. Fletcher, J. Altringham, J. Peakall, P. Wignall, R. Dorrell, Hydrodynamics of fossil fishes, in: Proc. R. Soc. B, volume 281, The Royal Society, 2014, p. 20140703. 
[6] J. Best, The fluid dynamics of river dunes: A review and some future research directions, Journal of Geophysical Research: Earth Surface 110 (2005).

[7] A. Arfaie, A. Burns, R. Dorrell, J. Eggenhuisen, D. Ingham, W. McCaffrey, Optimised mixing and flow resistance during shear flow over a rib roughened boundary, International Communications in Heat and Mass Transfer 58 (2014) 54-62.

[8] A. Arfaie, Numerical Modelling of the Influence of Lower Boundary Roughness on Turbulent Sedimentary Flows, Ph.D. thesis, University of Leeds, 2015.

[9] J. T. Eggenhuisen, W. D. McCaffrey, The vertical turbulence structure of experimental turbidity currents encountering basal obstructions: implications for vertical suspended sediment distribution in nonequilibrium currents, Sedimentology 59 (2012) 1101-1120.

[10] J. T. Eggenhuisen, W. D. McCaffrey, P. D. Haughton, R. W. Butler, Small-scale spatial variability in turbidity-current flow controlled by roughness resulting from substrate erosion: field evidence for a feedback mechanism, Journal of Sedimentary Research 80 (2010) 129-136.

[11] J. T. Eggenhuisen, W. D. McCaffrey, P. D. Haughton, R. W. Butler, Shallow erosion beneath turbidity currents and its impact on the architectural development of turbidite sheet systems, Sedimentology 58 (2011) 936-959.

[12] K. M. Straub, D. Mohrig, J. Buttles, B. McElroy, C. Pirmez, Quantifying the influence of channel sinuosity on the depositional mechanics of channelized turbidity currents: A laboratory study, Marine and Petroleum Geology 28 (2011) 744-760. 
[13] T. Tokyay, G. Constantinescu, E. Meiburg, Lock-exchange gravity currents with a high volume of release propagating over a periodic array of obstacles, Journal of Fluid Mechanics 672 (2011) 570-605.

[14] S. McLean, S. Wolfe, J. Nelson, Spatially averaged flow over a wavy boundary revisited, Journal of Geophysical Research: Oceans 104 (1999) 15743-15753.

[15] S. Feng, H. Ning, Computational simulations of blown sand fluxes over the surfaces of complex microtopography, Environmental Modelling \& Software 25 (2010) 362-367.

[16] D. R. Parsons, G. F. Wiggs, I. J. Walker, R. I. Ferguson, B. G. Garvey, Numerical modelling of airflow over an idealised transverse dune, Environmental Modelling \& Software 19 (2004) 153-162.

[17] S. Takahashi, M. Du, P. Wu, T. Maki, S. Kawashima, Three dimensional numerical simulation of the flow over complex terrain with windbreak hedge, Environmental modelling \& software 13 (1998) 257-265.

[18] S. J. Wakes, T. Maegli, K. J. Dickinson, M. J. Hilton, Numerical modelling of wind flow over a complex topography, Environmental Modelling \& Software 25 (2010) 237-247.

[19] Y. Yang, Y. Shao, Numerical simulations of flow and pollution dispersion in urban atmospheric boundary layers, Environmental Modelling \& Software 23 (2008) 906-921.

[20] E. Solazzo, X. Cai, S. Vardoulakis, Improved parameterisation for the numerical modelling of air pollution within an urban street canyon, Environmental Modelling \& Software 24 (2009) 381-388.

[21] A. Chu, R. C.-W. Kwok, K. Yu, Study of pollution dispersion in urban areas using computational fluid dynamics (cfd) and geographic infor- 
mation system (gis), Environmental Modelling \& Software 20 (2005) $273-277$.

[22] R. May, Sediment transport in pipes, sewers and deposited beds, Technical Report, HR Wallingford, 1993.

[23] P. J. Skipworth, S. J. Tait, A. J. Saul, Erosion of sediment beds in sewers: Model development, Journal of environmental engineering 125 (1999) 566-573.

[24] S. E. Coleman, J. J. Fedele, M. H. Garc 1' a, Closed-conduit bed-form initiation and development, Journal of Hydraulic Engineering 129 (2003) 956-965.

[25] ANSYS CFX Theory Guide, v14, Ansys Inc (2011).

[26] L. Djenidi, R. Elavarasan, R. Antonia, The turbulent boundary layer over transverse square cavities, Journal of Fluid Mechanics 395 (1999) 271-294.

[27] J. Cui, V. C. Patel, C.-L. Lin, Large-eddy simulation of turbulent flow in a channel with rib roughness, International Journal of Heat and Fluid Flow 24 (2003) 372-388.

[28] B. E. Launder, D. B. Spalding, The numerical computation of turbulent flows, Computer methods in applied mechanics and engineering 3 (1974) 269-289.

[29] A. E. Perry, W. H. Schofield, P. N. Joubert, Rough wall turbulent boundary layers, Journal of Fluid Mechanics 37 (1969) 383-413.

[30] J. Jimenez, Turbulent flows over rough walls, Annu. Rev. Fluid Mech. 36 (2004) 173-196. 
[31] B. C. Kneller, S. J. Bennett, W. D. McCaffrey, Velocity structure, turbulence and fluid stresses in experimental gravity currents, Journal of Geophysical Research: Oceans 104 (1999) 5381-5391.

[32] J. E. Simpson, Effects of the lower boundary on the head of a gravity current, Journal of Fluid Mechanics 53 (1972) 759768.

[33] J. B. Taylor, A. L. Carrano, S. G. Kandlikar, Characterization of the effect of surface roughness and texture on fluid flowpast, present, and future, International journal of thermal sciences 45 (2006) 962-968. 